\title{
A Distributed Optimization Algorithm for Energy of Wireless Sensor Networks Based on Potential Game
}

\author{
Lingfeng Shao $\mathbb{D}$, Junjie Yang, and Jicheng Fang \\ School of Electronics and Information Engineering, Shanghai University of Electric Power, Shanghai, 200000, China \\ Correspondence should be addressed to Lingfeng Shao; sysslf@126.com
}

Received 13 October 2019; Accepted 28 December 2019; Published 21 January 2020

Academic Editor: Giulia Grancini

Copyright (C) 2020 Lingfeng Shao et al. This is an open access article distributed under the Creative Commons Attribution License, which permits unrestricted use, distribution, and reproduction in any medium, provided the original work is properly cited.

\begin{abstract}
Recently, we have witnessed the rapid development of techniques on upgrading energy efficiency for wireless sensor networks (WSN). With the improvement of the detection range and the detection intensity, the lifetime of wireless sensor networks (WSN) is still limited by sensor node batteries (BA). Due to the need for wireless sensor network energy optimization, the power supply side has been putting forward higher requirements, and the traditional wireless sensor network with energy supplement has difficulty in meeting this development trend. The game and potential game concepts were introduced to take economics into account. Taking the wireless sensor network (WSN) with photovoltaic (PV) array charging and mobile-charging car (MCC) as an example, a running optimization model based on potential game is proposed, and the existence of Nash equilibrium has been proven. The iterative solution is completed by communication between the players, and the energy utilization rate is effectively improved. This paper verifies that potential game theory can be used to improve the feasibility and efficiency of wireless sensor network energy optimization.
\end{abstract}

\section{Introduction}

The wireless sensor network is a new emerging network technology composed of sensor nodes with sensing, computing, and communication in a self-organizing manner. The information of the object is perceived by the wireless sensor network through the collection network. It uses multihop as a wireless communication method to transmit the collected and processed information to the base station and finally to the end user. In the power industry, with the in-depth construction of the smart grid, the monitoring of the operational status of the equipment has been put forward in the power generation, transmission, substation, power distribution, power consumption, and other aspects of the power system. The requirement of monitoring appeared in power plants, which is based on wireless sensor networks. The optimization of energy strategy has become the primary consideration for sensor network design in wireless sensor networks, due to insufficient energy supplementation or unpredictable energy supplementation. It is effective to use wireless sensor network technology with multiple charging sources to solve the lifelimited problem. In a rechargeable power monitoring sensor network, nodes can be completely free of energy constraints if the nodes can acquire enough energy or the energy of the nodes can be replenished periodically and continuously. However, due to the weather, the supplement of node energy is unpredictable and unreliable, in many solar and wind power applications.

Therefore, this paper believes that the operation of the wireless sensor network needs to be equipped with MCCs in the case of PV supplementation.

Operational optimization is the core approach to efficient energy management. The optimization problem of wireless sensor network energy is a multiobjective collaborative optimization problem, including economic, technical, and environmental factors.

Considering energy saving, the routing protocols are used to reduce the overall energy consumption of wireless sensor network node [1-4]. Kim and Kim focused that under the signal-to-interference-plus-noise ratio constraint, optimum energy-saving strategies are introduced based on common and adaptive transmit power [5]. The routing algorithm with sleep mode is used in wireless sensor network to improve lifetime [6]. From the point of view of charging, the path 


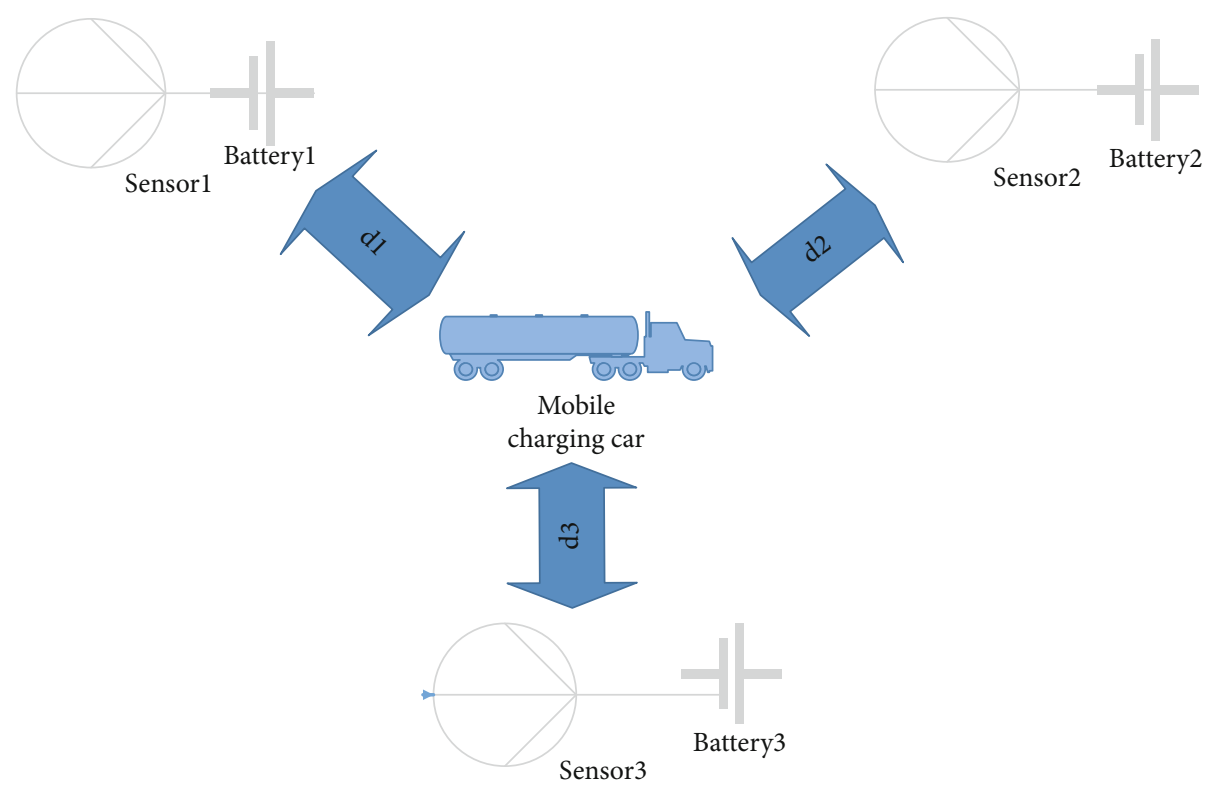

FiguRE 1: Structure of wireless sensor network.

planning problem of MCCs is also used for long-term survival of WSN, considering uncertainties like PV energy [7-9]. Rao et al. take the effects of charging distance and angle on charging efficiency into consideration [7]. Considering the research of charging and energy saving is very helpful for the development of WSN. Currently, sleep scheduling [10], wireless charging [11, 12], etc. are considered. However, some methods require that the energy replenishment process be independent or distributed or Markov processed, and the energy source is insufficient to adapt to the high-consumption WSN.

High-energy WSN and sustainable development are the points of consideration in this article. Game theory in wireless sensor networks is mainly used for network coverage [13], information transmission [14, 15], etc., but is less used for energy optimization. Recently, the research of potential game had been deepened by Monderer and Shapley [16]. It provides an efficient method for optimization in multiplayer systems where each player is self-optimizing and gets a Nash equilibrium. It has been successfully used in many places [17-19], including wireless sensor networks [20] and communication [21] The feature of this paper is as follows:

(1) A revenue model is established for the energy of the heterogeneous wireless sensor network from the perspective of economy

(2) Complex individual constraints are transformed into the strategy space of the players. From the perspective of the game, the potential game model is unified by the individual

(3) The strategy is solved by a distributed method, which fully reflects the individual's intelligence

The rest of this article is organized as follows: Section II describes the structure of the wireless sensor network and the basic concept of the potential game and determines a uniform revenue function. Section III analyzes the algorithm of wireless sensor network based on potential game and proposes an optimization method. Section IV shows the simulation results. Section V shows the conclusion.

\section{Wireless Sensor Network Model Based on Potential Game}

2.1. The Structure of Wireless Sensor Network. Relying on the laboratory sensor network system, a complete wireless sensor network is established by adding photovoltaic panels, leadacid BA and MCC. The structure is shown in Figure 1.The system is mainly composed of PV arrays, MCC, storage BA, and underlying equipment units and power controllers (which is composed of sensors), as well as top-level energy management systems. The energy management system is the core of the network to coordinate and control the operation of the entire system, including human-computer interaction, prediction module, operation optimization, and scheduling control. Based on this, this paper mainly studies the autonomous operation optimization of wireless sensor networks with energy supplementation.

2.2. Game Theory and Potential Game. Game theory is a research of mathematical models of strategy interaction between rational decision-makers. It is used in all area of social science, as well as in logic and computer science. At the beginning, it addressed zero-sum games, in which one player's gains result in losses for the other players. Today, game theory applies to a wide range of behavioral relations and is now an umbrella term for the science of logical decision making in humans, animals, and computers [22]. The game is divided into cooperative games and noncooperative games according to whether or not a binding agreement can be reached. 
In game theory, the Nash equilibrium, named after the late mathematician John Nash, is a proposed solution of a noncooperative game involving two or more players. Each player knows the equilibrium strategies of the other players, and the other player has nothing to gain by changing only their own strategy.

The potential game is such a type of game. As a special form of noncooperative game, it has limited improvement characteristics, which was first proposed by Monderer and Shapely in 1996 [23].

2.2.1. Definition of Potential Game. The potential game [16] is divided into ordinal potential game, weighted potential game, and full-potential game. The full-potential game is used the most, and the model of this paper will be built according to the full-potential game.

Definition 1. For the game $\Gamma=\left\langle N,\left\{S_{i}\right\}_{i \in N},\left\{R_{i}\right\}_{i \in N}\right\rangle$, there exists a function $P: S \rightarrow P, \forall i \in N$ [24],

$$
R_{i}\left(r^{i}, r^{-i}\right)-R_{i}\left(r^{i \prime}, r^{-i}\right)=P\left(r, r^{-i}\right)-P\left(r^{i \prime}, r^{-i}\right),
$$

where game $\Gamma$ is a complete potential game, $N$ is a player, $S$ is the strategy of all players, $R$ is the revenue function of all players, $S_{i}$ is the strategy of $i^{\text {th }}$ player, $S^{-i}$ is the strategic combination of the remaining players except $i^{\text {th }}$ player, the strategy of all players can be expressed as $S=\left(s^{i}, s^{-i}\right)$.

\subsubsection{The Properties of the Potential Game}

(1) Attribute 1: the potential function of game $\Gamma=\langle N$, $\left.\left\{S_{i}\right\}_{i \in N},\left\{R_{i}\right\}_{i \in N}\right\rangle$ is $P: \bigotimes S \rightarrow P$; then, the Nash equilibrium of $\Gamma=\left\langle N,\left\{S_{i}\right\}_{i \in N},\left\{R_{i}\right\}_{i \in N}\right\rangle$ and the Nash equilibrium of $\Gamma=\left\langle N,\left\{S_{i}\right\}_{i \in N},\left\{R_{i}\right\}_{i \in N}\right\rangle$ are consistent

(2) Attribute 2: every finite-order potential game has a pure-strategy equilibrium solution

(3) Attribute 3: every finite ordinal potential game has finite improvement property

The Nash equilibrium must exist, if the game model based on the potential game satisfies the three attributes of the potential game. Therefore, we only need to prove that the model is a potential game, and the convergence of the model does not need to be proven again.

2.3. Game Modeling of Energy Individuals Based on Wireless Sensor Networks. This paper will map the wireless sensor network to the three elements of the game. The three elements are the players, the revenue function, and the strategy space. The specific instructions are as follows:

(1) The players: in order to fully embody the autonomy of each power source in the sensor network, each energy source is mapped to a player. The players in the bureau include photovoltaic arrays, MCCs, and $\mathrm{BA}$, and players can make decisions on their own. The players in the game maximize their own benefits in the process of the game, while promoting the optimal system benefits

(2) The revenue function: this paper coordinates the power processing in economic perspective

$$
\left\{\begin{array}{l}
R_{i}=E_{i}, \quad i \in N, \\
E_{i} \in\left\{E_{\mathrm{pv}}, E_{\mathrm{mcc}} E_{\mathrm{ba}}\right\}, \quad i \in N
\end{array}\right.
$$

(3) The strategy space: the strategy space of each source is constrained by the scope of the constraints of each energy source, so the strategy of the potential game is the output combination of photovoltaic array, mobile-charging trolley, and lead-acid battery

$$
\left\{\begin{array}{l}
S_{i}=\left\{\mathrm{P}_{i}: \mathrm{P}_{i . \min }<\mathrm{P}_{i}<\mathrm{P}_{i \cdot \max }\right\}, \\
P_{i} \in\left\{P_{\mathrm{pv}}, P_{\mathrm{mcc}}, P_{\mathrm{ba}}\right\}, \\
\quad i \in N
\end{array}\right.
$$

According to Attributes 2 and 3 of the potential game, it is necessary to linearly divide the strategy space (the discrete interval is determined according to the accuracy requirement, and currently, $1 \mathrm{~kW} / \mathrm{step}$ is recognized) to complete the discretization, making it a limited strategy space and ensuring that the game is a finite game. In addition, because they are heterogeneous, they cannot reciprocate the revenue function in a uniform form. It is necessary to separate the resumed revenue function for each individual and establish a strategy space.

\subsubsection{Photovoltaic Player}

(1) The revenue function: the revenue function of photovoltaic is mainly divided into two parts, the value of input photovoltaic array power generation and the costs of operation and maintenance [25], as shown in

$$
E_{\mathrm{pv}}=C_{\mathrm{ep}} P_{\mathrm{pv}} \Delta t-\gamma_{\mathrm{pv}} P_{\mathrm{pv}} \Delta t,
$$

where $E_{\mathrm{pv}}$ is the revenue of $\mathrm{PV}$ player, $C_{\mathrm{ep}}$ is the electricity price, $P_{\mathrm{pv}}$ is the power generation of $\mathrm{PV}$ array, and $\gamma_{\mathrm{pv}}$ is the loss factor of the PV array

(2) Prediction of PV input: the PV power characteristics [14] are as shown in

$$
P_{\mathrm{ppv}}=\frac{P_{\mathrm{STC}} G_{\mathrm{AC}}\left[1+k_{s}\left(T_{c}-T_{r}\right)\right]}{G_{\mathrm{STC}}},
$$

where $P_{\mathrm{ppv}}$ is the predicted power generation of PV player, $P_{\text {STC }}$ is the maximum test power under standard test conditions, $G_{\text {STC }}$ is the illumination under standard test conditions $\left(1 \mathrm{~kW} / \mathrm{m}^{2}\right), k_{s}$ is the temperature coefficient $\left(0.47 \% /{ }^{\circ} \mathrm{C}\right), T_{\mathrm{c}}$ is the operating temperature of $\mathrm{PV}$ array, and $T_{r}$ is the standard temperature $\left(25^{\circ} \mathrm{C}\right)$ 
(3) Strategy space

$$
S_{\mathrm{pv}}=\left\{P_{\mathrm{pv}}: 0 \leq P_{\mathrm{pv}} \leq P_{\mathrm{pv} \cdot \max }\right\}
$$

where $P_{\text {pv.max }}$ is equal to the PV-predicted output power in equation (5).

\subsubsection{Mobile-Charging Car (MCC) Player}

(1) The revenue function: the revenue function of MCC is expressed as follows:

$$
\begin{aligned}
E_{\mathrm{mcc}}= & \left(C_{\text {ep }} P_{\text {mcc-system }}-C_{\text {ep }} P_{\text {mcc-out }}-\gamma_{\text {mcc }} P_{\text {mcc-system }}\right) \Delta t \\
& -D_{\text {move }}
\end{aligned}
$$

where $E_{\mathrm{mcc}}$ is the revenue function of the mobile-charging car, $P_{\text {mcc-system }}$ is the power that the mobile-charging car actually charges the system, $P_{\text {mcc-out }}$ is the output power of the mobile-charging car, $\gamma_{\mathrm{mcc}}$ is the operation and maintenance cost coefficient of the mobile-charging car, and $D_{\text {move }}$ is mobile-charging car running cost.

(2) Strategy space: the output length of the MCC must meet the requirements of its running time, expressed as follows:

$$
\begin{gathered}
\left\{\begin{array}{l}
0 \leq t \leq t_{\mathrm{max}}, \\
t_{\mathrm{max}}=\frac{E_{\text {max }}}{P_{\mathrm{mcc}-\mathrm{in}}}
\end{array}\right. \\
S_{\mathrm{mmc}}=\left\{P_{\mathrm{mcc}}: 0 \leq P_{\mathrm{mcc}} \leq P_{\mathrm{mcc}-\mathrm{in}}\right\}
\end{gathered}
$$

\subsection{Lead-Acid Battery Player}

(1) The revenue function: the revenue function of leadacid BA is expressed as follows:

$$
E_{\mathrm{ba}}=C_{\mathrm{ep}} P_{\mathrm{ba}} \Delta t-\gamma_{b a}\left|P_{b a}\right| \Delta t
$$

where $E_{\mathrm{ba}}$ is the revenue of the battery, $P_{\mathrm{ba}}$ is the charge and discharge power of the battery, considered to be discharged when $P_{\mathrm{ba}}>0$, and $\gamma_{\mathrm{ba}}$ is the operation and maintenance cost coefficiency of the battery.

(2) The model of charge and discharge: the relationship between the charge and discharge powers $\mathrm{A}$ and $\mathrm{B}$ of the lead-acid battery and the state of charge is as follows [25]:

$$
\begin{aligned}
& \operatorname{SOC}(t)=\operatorname{SOC}(t-1)(1-\delta)+\frac{P_{c} \Delta t \eta_{c}}{E_{r}}, \\
& \operatorname{SOC}(t)=\operatorname{SOC}(t-1)(1-\delta)+\frac{P_{d} \Delta t}{E_{r} \eta_{d}},
\end{aligned}
$$

where $\operatorname{SOC}(t)$ is the state of charge of the battery at time $t ; P_{c}, P_{d}, \eta_{c}$, and $\eta_{d}$ are, respectively, the charging power of the battery, the discharging power of the battery, and the charging and the discharging efficiency of the battery, $E_{r}$ is the rated capacity of the battery, and $\delta$ is the self-discharge rate of the battery.

(3) Strategy space: because the battery charge and discharge power are limited, we take the battery charge and discharge per unit time that does not exceed $20 \%$ of its rated capacity, expressed follows:

$$
\left\{\begin{array}{l}
\frac{P_{c} * 1 h \leq 20 \% E_{r}}{\eta_{c}}, \\
P_{d} * 1 h \leq 20 \% E_{r} \eta_{d}
\end{array}\right.
$$

This paper ensures that the battery has sufficient realtime scheduling margin and emergency power support without PV replenishment at night, where $\mathrm{SOC}_{\min }$ is the minimum charge of the battery. The maximum charge of the battery is set to $\mathrm{SOC}_{\max }$ to prevent overcharging of the battery, expressed follows:

$$
\mathrm{SOC}_{\min } \leq \mathrm{SOC} \leq \mathrm{SOC}_{\max }
$$

The allowable charge and discharge power of the battery are constantly changing with the state of charge, expressed follows:

$$
\begin{aligned}
P_{c} & =\frac{\min \left(P_{c \cdot \max },\left(\mathrm{SOC}_{\max }-\mathrm{SOC}\right) E_{r}\right)}{\eta_{c}}, \\
P_{d} & =\min \left(P_{d \cdot \max },\left(\mathrm{SOC}-\mathrm{SOC}_{\min }\right) E_{r} \eta_{d}\right),
\end{aligned}
$$

where $P_{c \cdot \max }$ and $P_{d . \max }$ are both positive values.

$$
S_{\mathrm{ba}}=\left\{P_{\mathrm{ba}}:-P_{c} \leq P_{\mathrm{ba}} \leq P_{d}\right\}
$$

2.5. Energy Potential Game Model and Analysis of Wireless Sensor Networks. The heterogeneity of energy individuals in wireless sensor networks makes no uniform representation of both the income function and the strategy space. Determining the revenue function and finding the potential function is the key to issue potential game modeling. In order to maximize the benefits of each power source in the game process while optimizing the efficiency of the system, consider constructing the potential function as the sum of the benefits of all players, expressed follows:

$$
W(y)=\sum_{i}^{N} R_{i}=\sum_{i}^{N} E_{i} .
$$

The wireless sensor network game model established by equation (17) is a complete potential game. From the definition of the potential game, the proof process is as follows: 


$$
\begin{gathered}
W(s)=W\left(s^{i}, s^{-1}\right)=E_{i}+\sum_{j, j \neq i}^{N} E_{j} . \\
\Delta W=W\left(s^{i}, s^{-i}\right)-W\left(s^{i \prime}, s^{-i}\right) \\
=\left(E_{i}+\sum_{j, j \neq i}^{N} E_{j}\right)-\left(E_{i}^{\prime}+\sum_{j, j \neq i}^{N} E_{j}\right) \\
=E_{i}-E_{i}^{\prime} . \\
\Delta R=\mathrm{R}\left(s^{i}, s^{-i}\right)-\mathrm{R}\left(s^{i \prime}, s^{-i}\right)=E_{i}-E_{i}^{\prime} . \\
\Delta W=\Delta R .
\end{gathered}
$$

From equation (21), the game model established on the model of the energy operation of the wireless sensor network satisfies the definition of the complete potential game and has all the attributes of the potential game. After the discretization of the strategy space, the potential game is a finite potential game, so it has a finite improvement characteristic. From Attribute 2, it can be known that the potential game must have a pure strategy Nash equilibrium solution.

\section{Analysis of Distributed Optimization Algorithm Based on Potential Game}

This paper studies the distributed optimization of wireless sensor network energy supply and chooses the optimization from the utility function of the game. It is a way to match the potential game with the distributed. In the framework of the potential game, it can not only maximize the benefits of players but also optimize the system. This paper's potential game optimization is mainly divided into the following parts:

(1) determination of strategy space

(2) policy evaluation and decision rules

(3) policy update

(4) power balance

3.1. Determination of Strategy Space. The constraints are different for each player. In order to adapt to the requirements of the game, the constraints must be transformed into the strategy space of the players, simplifying the game model.

The corresponding maximum allowable output power is determined to be calculated by the photovoltaic array according to the power prediction model and environmental parameters. The photovoltaic strategy is the discrete data points within the maximum allowable output power range.

The battery should update the state of charge, and consider the safe operation to determine the charge and discharge power allowed in the next period, and discretize it as the strategy space of the battery player.

The MCC should be considered to prevent overcharging of the battery within the basic power output range, and since the moving distance is negligible compared to the amount of charging, it will be ignored in later simulation.
3.2. Strategy Evaluation and Decision Rules. It is assumed that each player is individually rational; that is, the purpose of each player in the game is to maximize their own benefits. Therefore, it is the most direct way to use the revenue function of the player as the objective function of the strategy evaluation. The decision rule is adopted; that is, the strategy of maximizing the income function is selected as the next strategy. In the discrete strategy space, the players determine the optimal strategy through exhaustive.

3.3. Policy Update. After the players determine the optimal strategy, the policy updates are performed in a determined priority order. The PV array player has low operation and maintenance cost, and the photovoltaic energy is renewable energy, so it is updated first. The battery player plays the role of power balance and peak clipping in the sensor energy network, so the policy update sequence is set to the last. The revenue function of MCC is worse than PV, and environmental protection is not as good as PV; therefore, the order of policy updates is PV arrays, MCCs, and batteries. Players are assigned priorities based on the update order, and players can communicate with each other to ensure sequential updates.

3.4. Power Balance. Unlike other models, this potential game model must satisfy real-time power-balance constraints. Therefore, the penalty function is used to consider the power-balance constraint, and the power deficiency is set to characterize the degree of constraint satisfaction. The game model after balancing power is still considered a fullpotential game model, and the final penalty function term will approach 0 , and the resulting Nash equilibrium satisfies the power-balance.

3.5. Distributed Optimization Process. The distributed structure of the players is shown in Figure 2, and the specific process is shown in Figure 3.

In this algorithm, each player can communicate with each other to understand the decision-making situation of other players, and they have certain autonomy to maximize their own revenue. In the end, under the framework of the potential game, the common goal of optimizing system benefits can be realized.

\section{Simulation}

4.1. Determination of Strategy Space. A simulation was conducted for a wireless sensor network consisting of three groups of PVs, three MCCs, three BA, and three sensor nodes (for the convenience of the example simulation, this paper concludes that the moving time of MCC can be neglected, regardless of charging loss, and the energy of each PV arrays and BA can circulate among the three sensor nodes.) The coefficients of maintenance cost of different equipment are shown in Table 1 [26, 27]. The environmental parameters for $48 \mathrm{~h}$ including temperature and light intensity are shown in Figure 4. The parameters of photovoltaic arrays are shown in Table 2 (CHSM6610P/HV array). The parameters of BA are shown in Table 3 [25]. The given parameters of the mobile-charging car are listed in Table 4 (laboratory- 


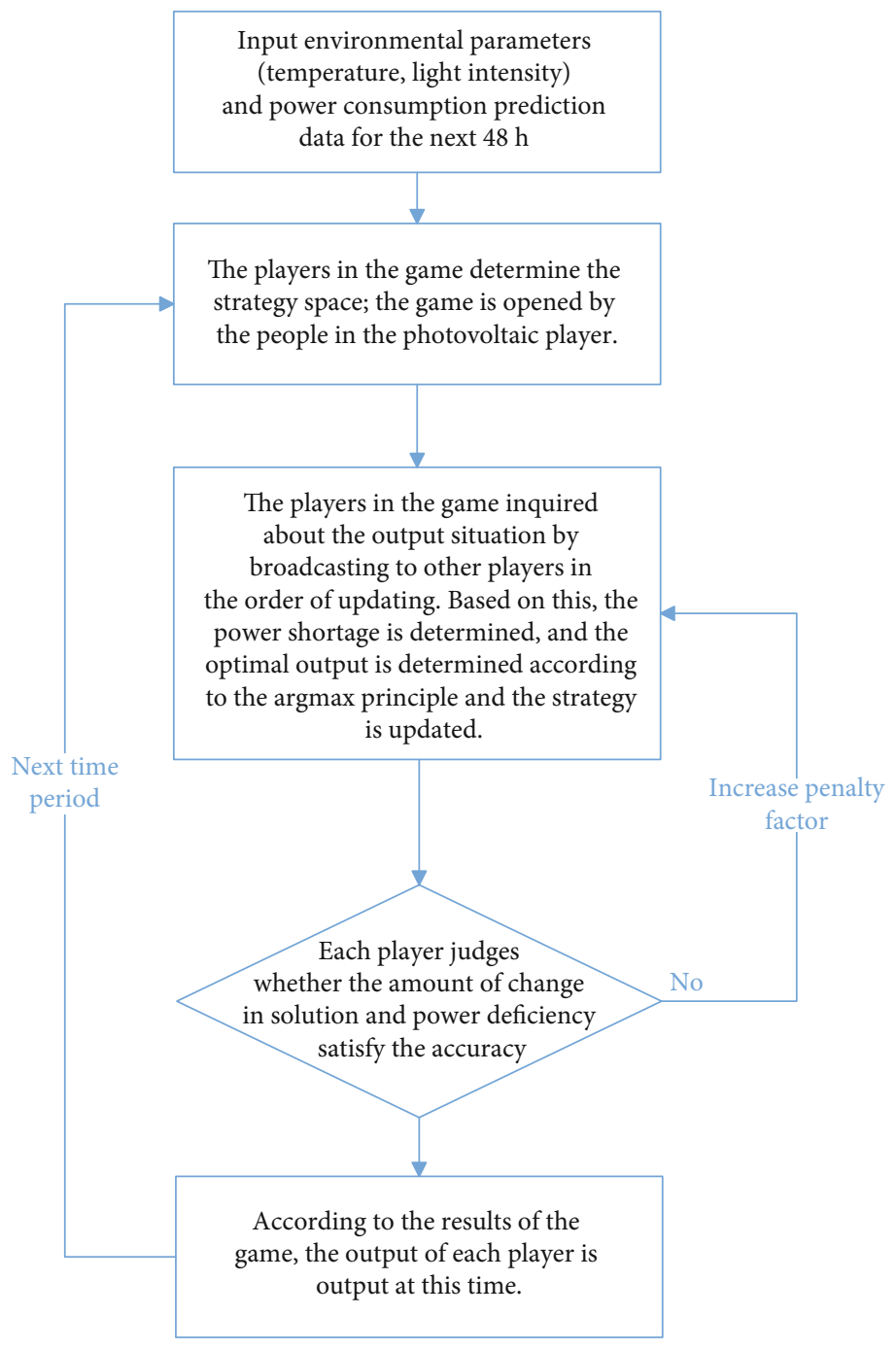

Figure 2: The flow chart of game iteration.

measured data). The prediction of maximal power generation of PV array and power usage of wireless sensor networks are shown in Figure 4 (in order to better verify this method, this paper selects 48 hours of simulation and assumes that the situation on the second day is exactly the same as the first day.) Three typical conditions are considered: (1) adequate PV supplements (10:00-16:00); (2) insufficient PV supplementation $(0: 00-10: 00)$ and $(16: 00-23: 00)$; and (3) the second day's phenomenon is similar to the first day.

4.2. Analysis of Simulation Results. Figure 5 shows that the load forecast has peaks and valleys, and the peak appears between 8:00 and 16:00. The predicted peak output of PV also appears in this period, which fits the load demand to a certain extent. However, BA and MCCs are still required to provide corresponding energy support. During the $0: 00 \sim 8: 00$ period and 17:00 23:00 period, the load occasionally has a small peak, but at this time, there is no light and the PV array output is zero; to satisfy the load demand, the MCC should be used when necessary. The BA assist in ensuring power balance.
Through experimental simulation, the potential game optimization results of PVs, MCCs, and BA are shown in Figures 6-9. It can be seen from Figure 6 that the PV game output coincides with the maximum predicted output (except 12:00), which fully reflects the priority of PV output. Figure 7 shows the SOC and charge and discharge curves. Figure 8 shows the SOC without the MCC; Figure 9 shows the SOC without the method. Figure 10 shows the output power of sleep strategy and predicted load. Figure 11 shows the SOC with greedy selection strategies, based on sleep mode switching.

From Figures 6 to 9, the results mean that the game is reasonable and valid. In the past, some scholars believe that the dormancy strategy can guarantee the survival of WSN. Figures 10 and 11 show that the existing high-power WSN cannot guarantee this method [28]. The characteristics can be summarized as follows.

Figure 6 shows that the output of the PV game coincides with the maximum predicted output (except 12:00), while the predicted output is higher than the rated power. In the first day from 10:00 to 15:00 and the second day from 


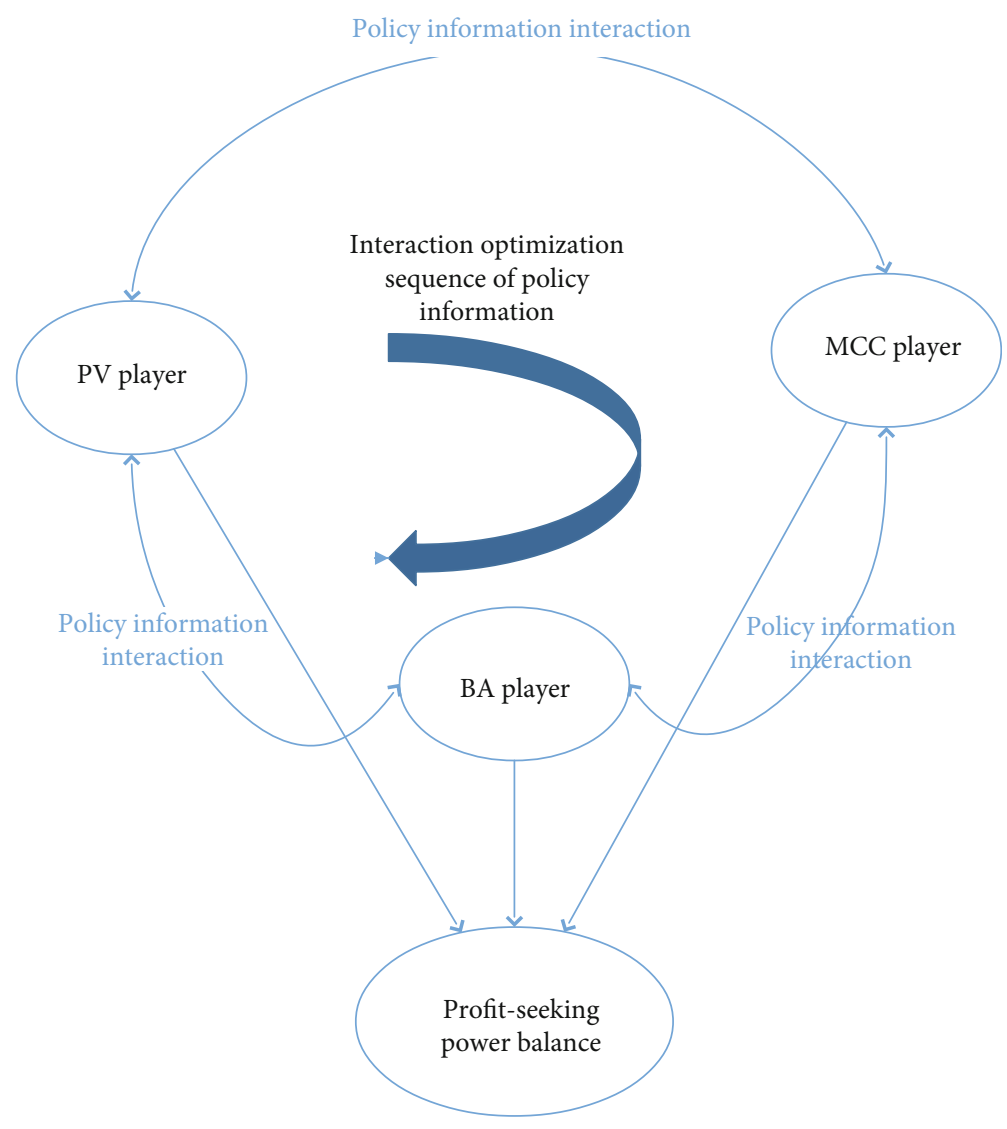

FIGURE 3: Distributed structure of players.

TABLE 1: Coefficient of maintenance cost of different equipment.

\begin{tabular}{lc}
\hline Equipment type & Maintenance cost $(\$ / \mathrm{kW})$ \\
\hline Photovoltaic array & 0.001396 \\
Battery pack & 0.026641 \\
Mobile-charging car & 0.001998 \\
\hline
\end{tabular}

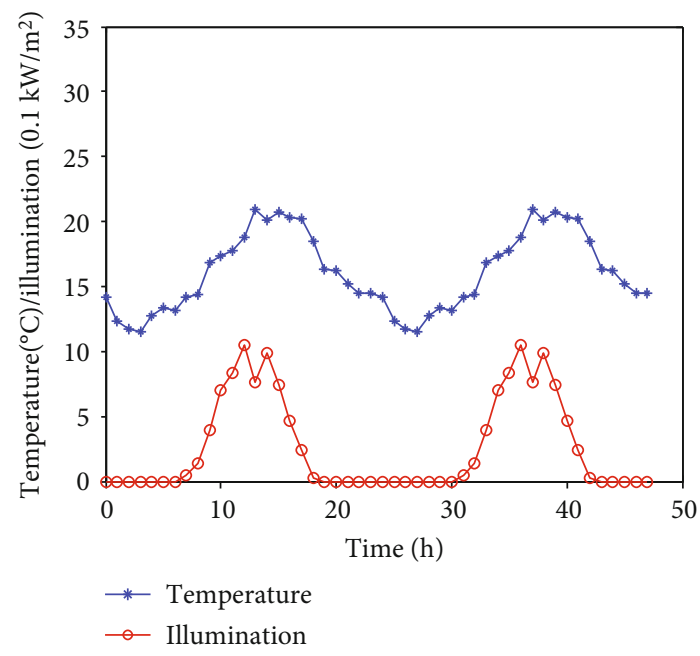

FIgURE 4: Date of the forecast $48 \mathrm{~h}$.
TABle 2: Photovoltaic array parameters.

\begin{tabular}{lc}
\hline Photovoltaic array & Rated power \\
\hline No. 1 & $2.75 \mathrm{~W}$ \\
No. 2 & $2.80 \mathrm{~W}$ \\
No. 3 & $2.85 \mathrm{~W}$ \\
Total & $8.4 \mathrm{~W}$ \\
\hline
\end{tabular}

TABLE 3: Battery parameters.

\begin{tabular}{lc}
\hline Parameters & Numerical value \\
\hline Total capacity $(\mathrm{kW} \cdot \mathrm{h})$ & 0.5 \\
Charge and discharge efficiency (\%) & $85 \%$ \\
Initial storage capacity & 0.25 \\
SOC $_{\text {min }}$ & 0.2 \\
\hline
\end{tabular}

TABle 4: Mobile-charging car parameters.

\begin{tabular}{lc}
\hline Parameters & Numerical value \\
\hline Total capacity $(\mathrm{kW} \cdot \mathrm{h})$ & 10 \\
Movement speed $(\mathrm{m} / \mathrm{s})$ & 1.8 \\
Power consumption $(\mathrm{W})$ & 25
\end{tabular}




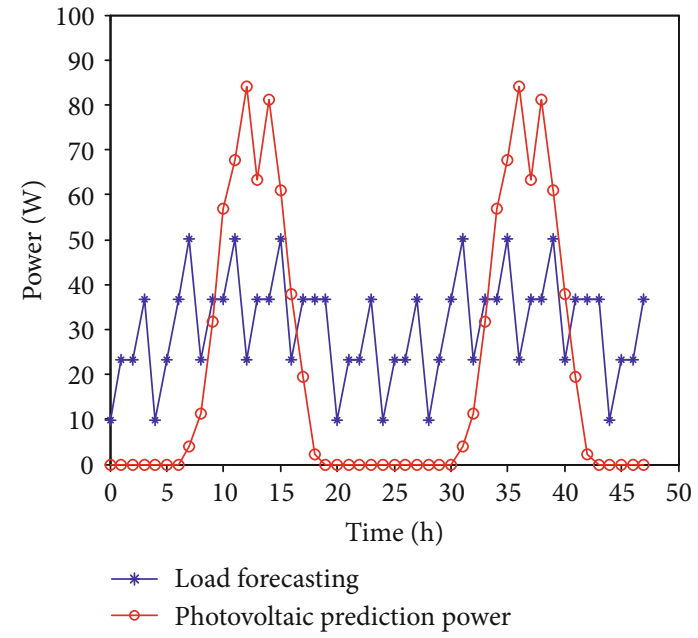

Figure 5: PV and load output power forecasting.

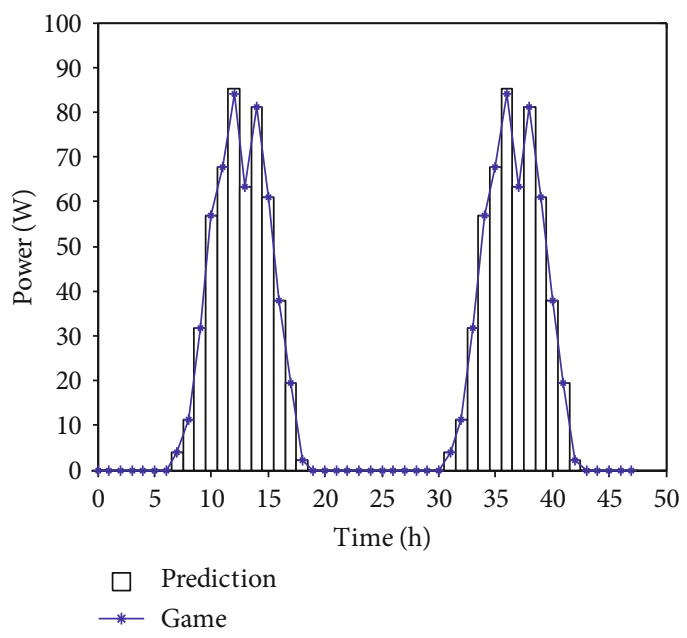

FIGURE 6: Comparison of optimal power generation and maximal output power of PV.

$10: 00$ to $15: 00$, the PV array generates much more electricity than the load, and the PV array charges the battery. The result of the game shows that while the power generation of the PV array is less than the power consumption of the load, the PV array can be used to maximize utilization. The PV array first provides battery charging and then adjusts power when the power generation of the PV array is more than the power consumption of the load.

Figure 7 shows the SOC of battery and its charge power and discharge power at different times. It is common to alternate the charging and discharging of the battery. When the load is low, the battery charges and absorbs the excess power from PVs and discharges at the peak load, which is in line with the economic operation of the battery. The whole SOC is above $20 \%$, which satisfies the setting of the minimum state of charge, effectively preventing the damage of the battery caused from excessive discharging and ensuring that the battery has sufficient margin for emergency situations. The charge and discharge power of the battery is limited to the
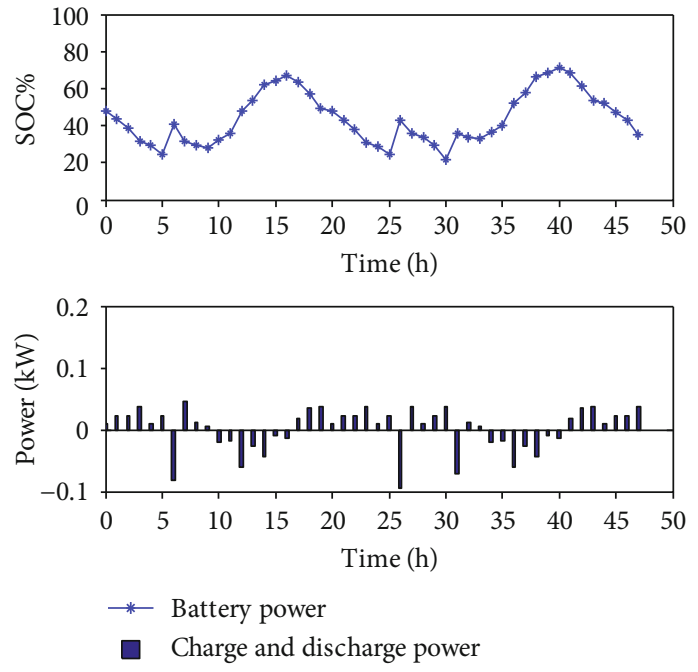

FIgURE 7: Battery charge state and $48 \mathrm{~h}$ charge and discharge power (the $\mathrm{BA}$ are charging while power $<0$; the $\mathrm{BA}$ are discharging while power $>0)$.
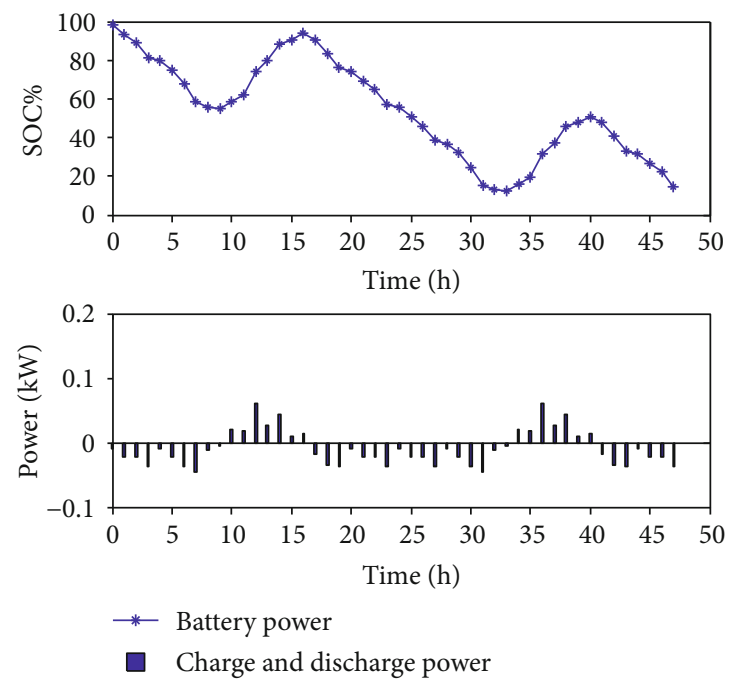

FIGURE 8: Battery charge state and $48 \mathrm{~h}$ charge and discharge power (without MCT).

maximum allowable charge and discharge power range, and the effect of the remaining power on the charge and discharge of the battery can be seen. As the SOC rises, the allowable charging power is reduced, effectively preventing the battery from overshooting. The game results are in line with it.

The game result of the MCC player is shown in the spikes of Figure 7. The charging at 6 o'clock on the first day, 2 o'clock on the next day, and 7 o'clock on the next day shows that the mobile car is not economically charged. The charging time of MCC is limited by the transportation consumption and the loss of charge and discharge.

Figure 8 shows the energy variation of PV WSN without MCC. Due to the large consumption, WSN cannot survive for a long time. 

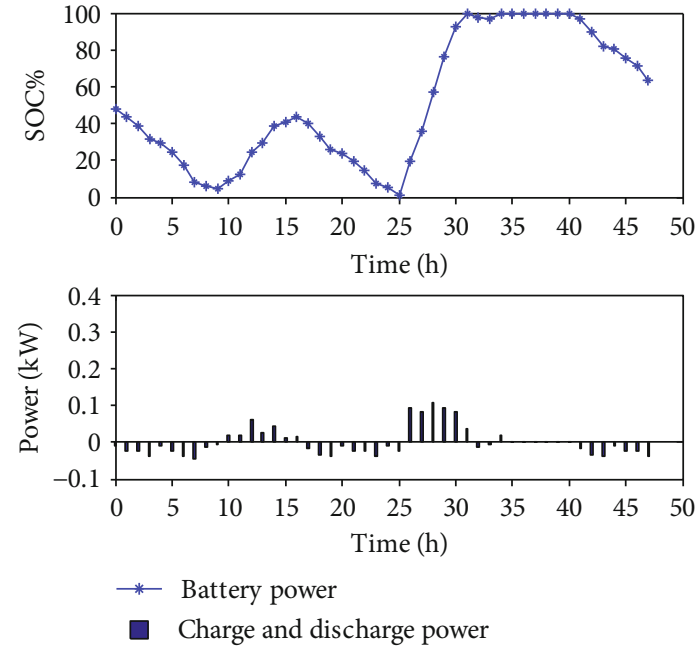

FIGURE 9: Battery charge state and $48 \mathrm{~h}$ charge and discharge power (without algorithm).

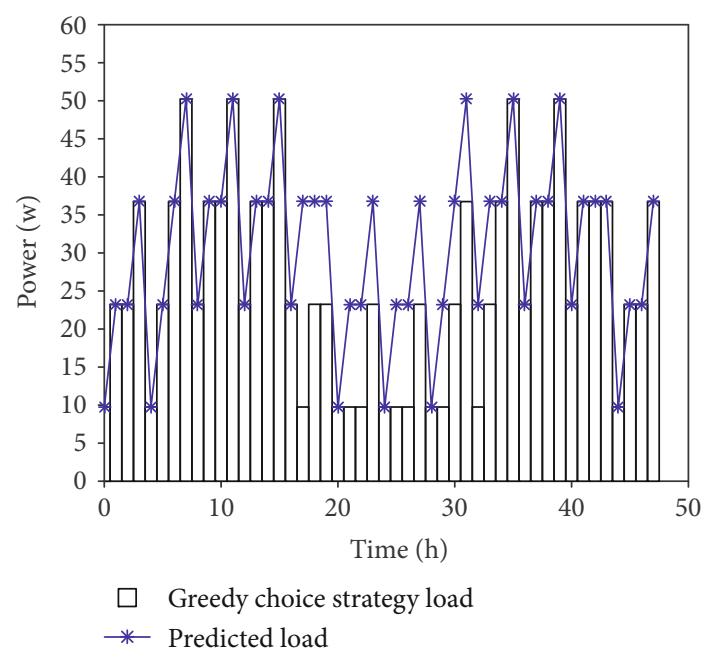

FIgURE 10: Output power of sleep strategy and predicted load (maximize energy usage with greedy selection strategies--based on sleep mode switching).

Figure 9 shows the energy variation of WSN without potential game. A SOC $>80 \%$ appeared on the second day from $6: 00$ to $20: 00$, and there was a large amount of abandonment from 10:00 to 16:00 on the next day. The above results and analysis fully demonstrate the feasibility and effectiveness of the potential game in the operation optimization of wireless sensor networks with PV and MCC.

Figures 10 and 11 show previous scholars' opinions that they believe that a suitable power consumption strategy can guarantee long-term operation of WSN. They use the greedy selection algorithm to change the dormancy strategy to fit the load curve, as much as possible to ensure survival before the next day. But the reality is that the energy of the battery gets less and less, so that it cannot fit the load very well. This means that the timeliness of the wireless sensor network is greatly reduced, which makes us regret. Compared with the WSN energy in the case of potential game, we can find that
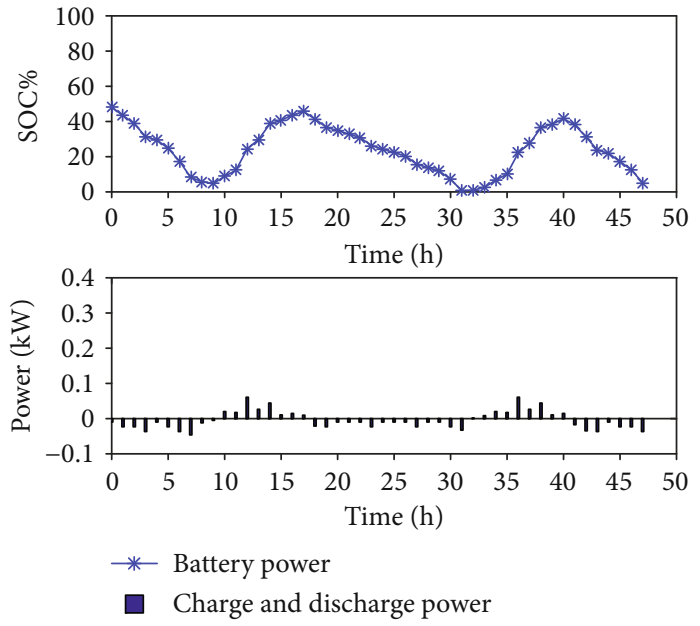

FIgURE 11: Battery charge state and $48 \mathrm{~h}$ charge and discharge power (maximize energy usage with greedy selection strategies_- based on sleep mode switching).

this algorithm can better guarantee the high-quality survival of WSN, which is of great significance.

\section{Conclusion}

(1) This paper establishes revenue models for heterogeneous sensor network energy modules and models them according to their respective characteristics from an economic perspective. The photovoltaic, MCC, and battery are mapped into players. The potential function is constructed by the player's income function, and the potential game model is proven

(2) This paper establishes a unified form of strategy space for the players by transforming the constraints into strategy space

(3) The potential game model is optimized by a distributed method, and the example is verified. The experimental results show that the players composed of PV, MCC, and BA have sufficient autonomy and independent decision-making ability. At the same time, the priority of PV promotes the highest utilization rate of renewable energy. The fast response feature of MCC ensures reliable power supply to the WSN; BA can promote the preservation of excess light energy and maintain the power balance during the game. The experiment proves that the distributed optimization method based on potential game can better protect the battery from overcharging and nondischarging and consume as much renewable energy as possible. The feasibility and effectiveness of the method presented in this paper has been proven

\section{Data Availability}

The specific parameters of photovoltaic power generation are based on the test results of electrical engineering laboratory 
of ZheJiang University in China. The economic parameters of batteries and mobile-charging car used to support the findings of this study are included within the article (Tables 1, 2, and 3).

\section{Conflicts of Interest}

The authors declare that there is no conflict of interest regarding the publication of this paper.

\section{Acknowledgments}

The author would like to thank the support of the Hongqiao Deng in School of Electrical Engineering, Zhejiang University, for photovoltaic panel experimental data and Linxun Liang for the mobile-charging trolley experimental data. This research was funded by the National Natural Science Foundation of China (61202369), Shanghai Technology Innovation Project (17020500900), and "Shuguang Program" sponsored by Shanghai Education Development Foundation and Shanghai Municipal Education Commission (17SG51).

\section{References}

[1] J. L. Li and D. P. Liu, "An energy aware distributed clustering routing protocol for energy harvesting wireless sensor networks," in 2016 IEEE/CIC International Conference on Communications in China (ICCC), pp. 1-6, Chengdu, China, July 2016.

[2] J. Yan, M. Zhou, and Z. Ding, "Recent advances in energyefficient routing protocols for wireless sensor networks: a review," IEEE Access, vol. 4, pp. 5673-5686, 2016.

[3] W. Huang, Y. Peng, J. Wen, and M. Yu, "Energy-efficient multi-hop hierarchical routing protocol for wireless sensor networks," in 2009 International Conference on Networks Security, Wireless Communications and Trusted Computing, pp. 469-472, Wuhan, Hubei, China, April 2009.

[4] A. Chunawale and S. Sirsikar, "Minimization of average energy consumption to prolong lifetime of wireless sensor network," in 2014 IEEE Global Conference on Wireless Computing \& Networking (GCWCN), pp. 244-248, Lonavala, India, December 2014.

[5] H. Kim and J. Kim, "Energy-efficient resource management in wireless sensor network," in 2011 IEEE Topical Conference on Wireless Sensors and Sensor Networks, pp. 69-72, Phoenix, AZ, USA, January 2011.

[6] G. Pradeebaa and N. Lavanis, "Network lifetime improvement using routing algorithm with sleep mode in wireless sensor network," in 2016 International Conference on Wireless Communications, Signal Processing and Networking (WiSPNET), pp. 1572-1575, Chennai, India, March 2016.

[7] X. Rao, P. Yang, Y. Yan, H. Zhou, and X. Wu, "Optimal recharging with practical considerations in wireless rechargeable sensor network," IEEE Access, vol. 5, pp. 4401-4409, 2017.

[8] C. Hu, "Research on charging planning and schedulability in wireless rechargeable sensor networks," Ph.D. dissertation, Southeast University, Nanjing, 2015.

[9] S.-H. Chen, Y.-C. Chang, T.-Y. Chen et al., "Prolong lifetime of dynamic sensor network by an intelligent wireless charging vehicle," in 2015 IEEE 82nd Vehicular Technology Conference (VTC2015-Fall), pp. 1-5, Boston, MA, USA, September 2015.
[10] M. Mukherjee, Y. R. Lu, R. Matam, and N. Choudhury, "Energy trading in sleep scheduling for wireless rechargeable WSNs," in 2018 10th International Conference on Communication Systems \& Networks (COMSNETS), pp. 429-431, Bengaluru, India, January 2018.

[11] C. Wang, J. Li, Y. Yang, and F. Ye, "Combining solar energy harvesting with wireless charging for hybrid wireless sensor networks," IEEE Transactions on Mobile Computing, vol. 17, no. 3, pp. 560-576, 2018.

[12] P. Zhou, C. Wang, and Y. Yang, "Self-sustainable sensor networks with multi-source energy harvesting and wireless charging," in IEEE INFOCOM 2019 - IEEE Conference on Computer Communications, pp. 1828-1836, Paris, France, April 2019.

[13] B. Shahrokhzadeh and M. Dehghan, "A distributed gametheoretic approach for target coverage in visual sensor networks," IEEE Sensors Journal, vol. 17, no. 22, pp. 7542-7552, 2017.

[14] J. Zheng, H. Zhang, Y. Cai, R. Li, and A. Anpalagan, "Gametheoretic multi-channel multi-access in energy harvesting wireless sensor networks," IEEE Sensors Journal, vol. 16, no. 11, pp. 4587-4594, 2016.

[15] J. Ren, H. Zhang, H. Hu, F. Cheng, and Y. Qin, “Achievable throughput analysis and channel access in energy harvesting cognitive radio sensor network," IEEE Access, vol. 7, pp. 82277-82287, 2019.

[16] D. Monderer and L. S. Shapley, "Potential games," Games and Economic Behavior, vol. 14, no. 1, pp. 124-143, 1996.

[17] M. Ye and G. Hu, "Solving potential games with dynamical constraint," IEEE Transactions on Cybernetics, vol. 46, no. 5, pp. 1156-1164, 2016.

[18] D. González-Sánchez and O. Hernández-Lerma, "Dynamic potential games: the discrete-time stochastic case," Dynamic Games and Applications, vol. 4, no. 3, pp. 309-328, 2014.

[19] D. González-Sánchez and O. Hernández-Lerma, “A survey of static and dynamic potential games," Science China Mathematics, vol. 59, no. 11, pp. 2075-2102, 2016.

[20] A. Moragrega, P. Closas, and C. Ibars, "Potential game for energy-efficient RSS-based positioning in wireless sensor networks," IEEE Journal on Selected Areas in Communications, vol. 33, no. 7, pp. 1394-1406, 2015.

[21] S. Zazo, S. Valcarcel Macua, M. Sanchez-Fernandez, and J. Zazo, "Dynamic potential games with constraints: fundamentals and applications in communications," IEEE Transactions on Signal Processing, vol. 64, no. 14, pp. 3806-3821, 2016.

[22] R. Tang, S. Wang, and H. Wang, "Optimal power demand management for cluster-level commercial buildings using the game theoretic method," Energy Procedia, vol. 159, pp. 186$191,2019$.

[23] Q. Wang, J. Zeng, Z. Wang, and J. Liu, "Distributed gametheoretic interactive algorithm for microgrid optimization," in 2018 37th Chinese Control Conference (CCC), Wuhan, China, July 2018.

[24] T. Inohara, "Relational Nash equilibrium and interrelationships among relational and rational equilibrium concepts," Applied Mathematics and Computation, vol. 199, no. 2, pp. 704-715, 2008.

[25] J. Zeng, Q. Q. Wang, J. F. Liu, W. Lin, and H. F. Guo, “An operation optimization algorithm of microgrid based on potential game," Proceeding of the CSEE, vol. 37, no. 24, pp. 7195-7204, 2017. 
[26] C. D. Li, R. W. Chen, J. Zeng, and J. F. Liu, "Research on optimization algorithm of microgrid energy management system based on non-cooperative game theory," Power System Technology, vol. 40, no. 2, 2016.

[27] X. Han, H. Zhang, X. Yu, and L. Wang, "Economic evaluation of grid-connected micro-grid system with photovoltaic and energy storage under different investment and financing models," Applied Energy, vol. 184, pp. 103-118, 2016.

[28] M. Zhao, J. Li, and Y. Yang, "A framework of joint mobile energy replenishment and data gathering in wireless rechargeable sensor networks," IEEE Transactions on Mobile Computing, vol. 13, no. 12, pp. 2689-2705, 2014. 

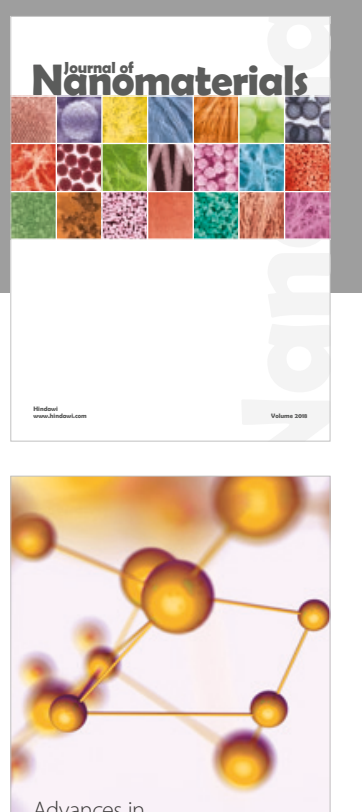

Physical Chemistry
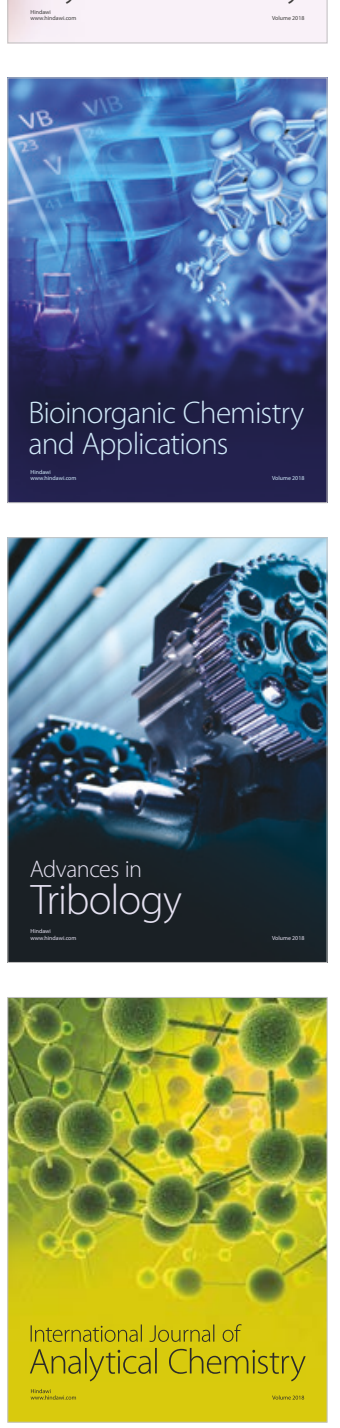

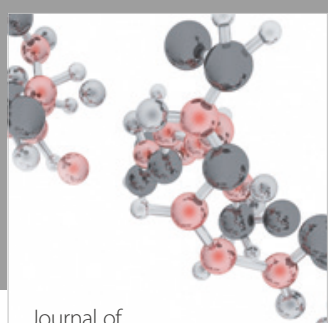

Analytical Methods

in Chemistry

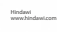

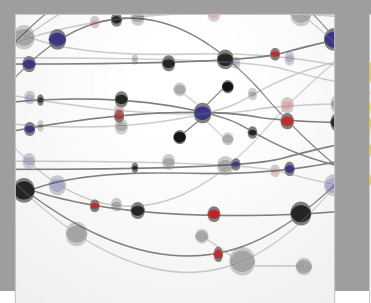

The Scientific World Journal

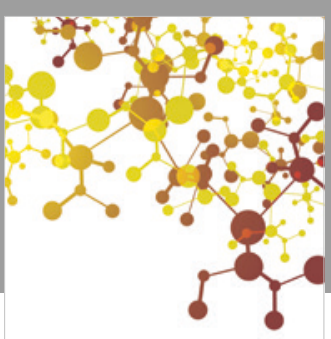

Journal of

Applied Chemistry
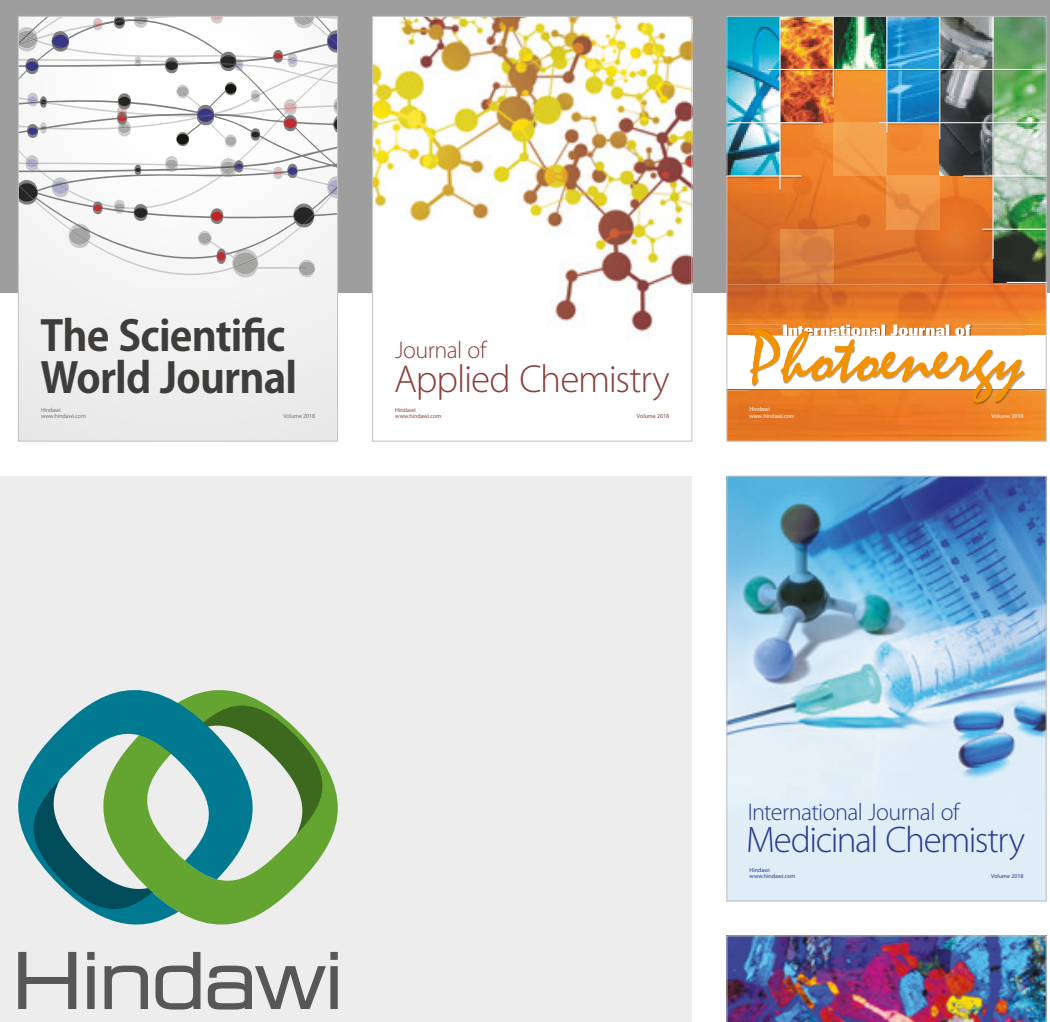

Submit your manuscripts at

www.hindawi.com
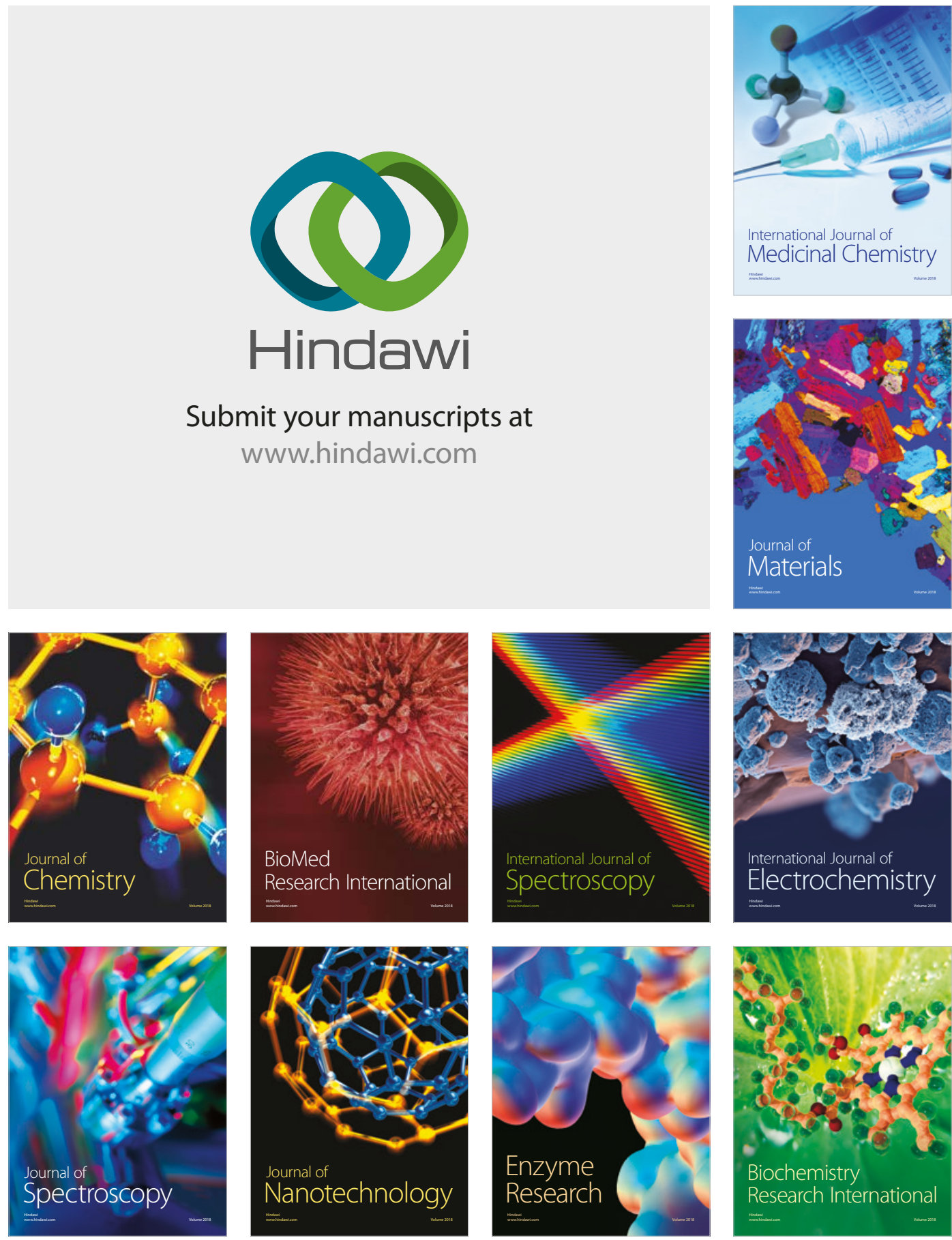
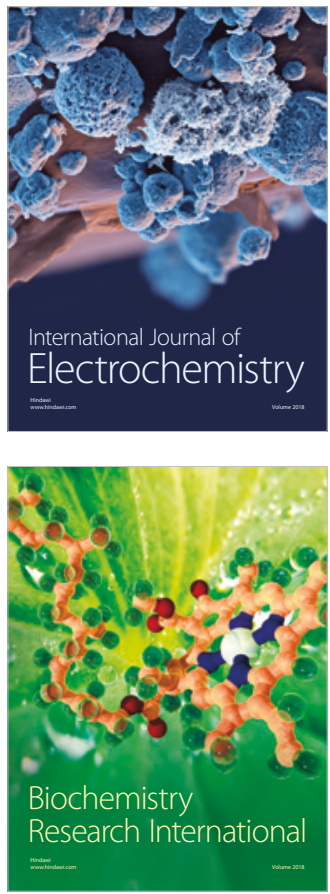